\title{
CUIDADO DOMICILIAR: PROPOSTA DE AÇÃO DA RESIDÊNCIA MULTIPROFISSIONAL EM SAÚDE DA FAMÍLIA - UFPEL
}

\author{
HOME CARE: PROPOSAL OF ACTION OF MULTIPROFISSIONAL RESIDENCE IN \\ FAMILY HEALTH - UFPEL
EL CUIDADO DOMICILIAR: LA PROPUESTA DE ACCIÓN DEL RESIDÊNCIA MULTIPROFISSIONAL EN LA SALUD DE LA FAMILIA -UFPEL

Rita Maria Heck*

Sidnéia Tessmer Casarin**

Adriana Damke Klock ${ }^{\star \star *}$

Luis Artur Rosa Filho****

\footnotetext{
* Enfermeira. Doutora em Enfermagem pela Universidade Federal de Santa Catarina. Prof. Adjunto FEO/UFPEL. Coordenadora Gerente da Residência Multiprofissional em Saúde da Família - UFPEL/Ministério da Saúde/BID.

** Enfermeira Residente. Residência Multiprofissional em Saúde da Família - UFPEL/Ministério da Saúde/BID.

*** Enfermeira Residente. Residência Multiprofissional em Saúde da Família - UFPEL/Ministério da Saúde/BID.

**** Médico Residente. Residência em Medicina Preventiva e Social UFPEL.
}

RESUMO. O cuidado domiciliar da saúde nas modalidades de um programa oficial de assistência publica é prática recente que vem tomando forma especialmente com o Programa de Saúde da Família (PSF). Este artigo descreve a proposta de ação no cuidado domiciliar, desenvolvido durante as atividades da Residência Multiprofissional em Saúde da Família/UFPEL-MS/BID ${ }^{* * * *}$. As discussões convergiram para o necessário embasamento teórico em relação à integralidade do cuidado à família e ao cuidado domiciliar em si. Acreditamos que esse artigo contribui para subsidiar a formulação de uma política em torno do tema "cuidado domiciliar", assim como orientar pesquisas na área.

PALAVRAS CHAVE: cuidado domiciliar; saúde da família; metodologia.

ABSTRACT. The home health care as an official public program is recent, and this modality is taking shape especially with Family Health Program (PSF). This article aims to describe a proposal of action in home care, developed during the Multiprofissional Residence at Pelotas Federal University - Brazil. The discussions improve a necessary theoretical support related to the integrality of care, in the family context and to the home care itself. We believe that this article could subsidize the politics programs on "home care", as well as guiding researches in this area.

KEYWORDS: home care; family health; methodology.

RESUMEN. El cuidado domiciliar de la salud en las modalidades de un programa oficial de asistencia publica es una reciente práctica que está tomando formas sobre todo con el Programa de Salud de la Familia (PSF). Este artículo describe una propuesta de acción en el cuidado domiciliar que se desarrolló durante las actividades de Residencia Multiprofesional en la Salud de la Familia/UFPELMS/BID. Las discusiones convergieron para el necesario embasamiento teórico sobre la integralidad del cuidado, respecto a la familia y al cuidado domiciliar en sí mismo. Nosotros creemos que este artículo puede servir con subsidios para la formulación de una política alrededor del tema "el cuidado domiciliar", así como guiando las investigaciones en el área.

PALABRAS-CLAVE: cuidado domiciliar; salud de la familia; metodología.

A Residência Multiprofissional Saúde da Família 2001-03 da Universidade Federal de Pelotas foi financiado pelo Banco Interamericano de Desenvolvimento (BID) através do Ministério da Saúde (MS).

Recebido em: 07/05/2004

Aceito em: 20/12/2004
Rita Maria Heck

Av. Duque de Caxias, 250 - Fragata

96030-002 - Pelotas - RS

E-mail: heck.sul@terra.com.br 


\section{INTRODUÇÃO}

O cuidado domiciliar da saúde nas modalidades de um programa oficial de assistência pública é uma prática recente que vem tomando forma especialmente com o Programa de Saúde da Família (PSF). Junto com esta política de ampliação dos serviços, preconiza novo modelo de assistência baseado nos princípios da universalidade, equidade e integralidade, em que se trabalhe saúde de forma ampla e compartilhada, valorizando-se tudo o que interage com o indivíduo, no contexto de sua experiência e pelas relações familiares e sociais que tão intensamente marcam sua realidade sanitária.

A inserção do Programa da Residência Multiprofissional em Saúde da Família em sintonia com a Residência de Medicina Preventiva e Social nas três Unidades Básicas de Saúde (UBS) da Universidade Federal de Pelotas repercutiu no processo de trabalho dos profissionais de saúde e trouxe mudanças nos serviços oferecidos aos usuários que viviam neste contexto. Assim, uma das ações em saúde que passou a fazer parte dos serviços é a assistência na modalidade de cuidado domiciliar.

Neste artigo descreveremos a proposta de ação em cuidado domiciliar, desenvolvido pelos profissionais que atuam no Programa de Residência Multiprofissional em Saúde da Família, da Universidade Federal de Pelotas.

\section{REFERENCIAL TEÓRICO}

Ao propor o projeto de cuidado domiciliar, achamos relevante retomar três conceitos que fazem parte do referencial teórico de compreensão desta discussão: a integralidade das ações de saúde, o cuidado e o cuidado em saúde da família.

\section{A INTEGRALIDADE NAATENÇÃO E NO CUIDADO À SAÚDE}

A questão do cuidado domiciliar está vinculada ao propósito de ampliação da integralidade das ações de saúde e à expansão da assistência prestada nas
Unidades Básicas de Saúde (UBS) para além destas, incluindo o domicílio. Assim, compreendemos integralidade como um conjunto de tendências cognitivas e políticas, especialmente atenção primária e promoção de saúde ${ }^{1}$.

Entretanto ao mesmo tempo que tentamos dar forma a um projeto que amplia a integralidade na atenção e no cuidado da saúde, como elemento estratégico que qualifica a saúde contemporânea, estamos diante de um desafio de vincular esta ao contexto histórico, social e político de vida no Brasil. Em outras palavras, significa a imprescindível tarefa de relativizar o tempo e o espaço, como elementos do contexto, para tecer um plano de cuidado voltado à integralidade da saúde da pessoa, sem perder de vista um projeto nacional maior de cidadania e descentralização das políticas públicas. Nessa dimensão, o que vem a ser integralidade do cuidado a saúde necessariamente passa pelas expectativas do usuário e deve incluir, também, como objetivo a cidadania como processo de emancipação e de promoção do autocuidado.

Faz parte desta proposta assistir para potenciar a autonomia do sujeito, a fim de preservá-lo da dor, do sofrimento, do envelhecimento e da morte precoce superando as deficiências fisico-biológicas, sentindose mais saudável, tendo alegria, disposição para a vida, recuperar o prazer das coisas cotidianas, estar com os outros (família, e amigos) rompendo com o isolamento social a saúde em oposição à morte social.

O desafio de promover uma assistência integral voltada para os objetivos do Sistema Único de Saúde (SUS) em que se idealiza uma nova prática assistencial, está vinculado à equipe de saúde. Esta equipe tem responsabilidade de construir e promover saúde junto com a comunidade, ocupando o espaço nas políticas públicas. Faz parte desta tarefa compreender que a família, sua vida privada e comunitária não estão isoladas, mas insertas na dinâmica política e econômica da sociedade como um todo e os conflitos sociais de classe, raça, sexo também devem ser abordados pelos trabalhadores da saúde, em sua prática profissional. Como desafio 
de atuar no espaço domiciliar está a necessidade de subsidiar e fortalecer relações familiares, respeitar vínculos afetivos e redes de solidariedade social específicos de cada local. Em síntese a intervenção do profissional deve estar voltada para construir educativamente valores de respeito à diversidade humana e nisso ampliar politicamente a autonomia do usuário/cidadão como elemento de relação social.

\section{A COMPREENSÃO DO CUIDADO EM SAÚDE}

O cuidado em saúde está vinculado à compreensão de que a doença não tem somente caráter físico-biológico; cumpre pensar a doença a partir de uma pluralidade de interpretações, divergências e negociações que envolvem os episódios de sofrimento do sujeito, até se chegar a um diagnóstico entre os sistemas locais de saúde. Esta percepção é compartilhada por Kleinman ${ }^{2}$, que entende que o cuidado está socialmente organizado, o que implica identificar os padrões de crença sobre causas, normas, relações de poder e locais em que se dão as interações. A partir deste referencial, idealiza um modelo em que relaciona três sistemas locais de cuidados à saúde: a) o setor popular, representado por pessoal não profissional relacionado ao doente, como família, vizinhança, amigos; b) o setor profissional, representado por profissões de cura organizadas, incluindo a homeopatia e a acupuntura; c) o setor folk, em que são reconhecidos especialistas de cura, porém sem burocracia e regulamentação, como as benzedeiras e as curandeiras ${ }^{2}$.

No cuidado como prática, um oficio, está vinculado à dimensão do tempo atual contemporâneo como afirmação da modernidade científica. No fim do século XIX, com o aparecimento de tecnologias elaboradas, o objeto do cuidado torna-se isolado, parcializado, separado das dimensões sociais e coletivas $^{3}$. Nessa perspectiva, cuidar passa a tratar a doença excluindo todas as outras concepções elaboradas no curso da história em face do problema da vida e da morte. A perspectiva de cuidado, nesta dimensão, segue a hipótese de “... tratar para erradicar o que pode fazer morrer, mas com o risco de deixar de olhar para tudo o que ainda vive, para aquilo que pode fazer viver, para aquilo que dá sentido à vida" 3 : 32 .

Os cuidados para a promoção da vida ${ }^{3}$ podem ser diferenciados em cuidados de reparação da vida (necessita ação de vigilância direta e contínua de profissional da saúde) e cuidados de manutenção da vida (poderão ser realizados pelo paciente ou pela família, seguindo orientações da equipe multiprofissional de saúde). Entendemos que esta diferenciação teórica indica a necessidade de objetivar focos distintos de organização do trabalho, sendo um direcionado a cuidados intensivos, e outro ao acompanhamento no contexto da família, comunidade, enfim no cuidado de atenção básica de saúde.

No cuidado domiciliar o profissional de saúde tem papel de mediador entre quem necessita de cuidados e a pessoa que vai realizar a ação do cuidado. Esta tarefa de mediador se acentua, pois o profissional de saúde não deve enfocar somente o que se passa no corpo físico de quem recebe cuidados, mas também é igualmente importante identificar quem pode assumir ou assume o cuidado na ausência do profissional. Assim, além de constatar sinais clínicos, o profissional necessita ver, interpretar e identificar a rede social a que o paciente pertence.

Neste cenário, a família passa a ser atriz que, além de interagir e definir padrões de cuidado, tem participação na definição da saúde, perspectiva esta que o profissional não deve perder de vista e, diante das mais adversas situações, ter presente que neste programa se tem a necessidade de fazer um caminho inverso em que o objetivo é fortalecer os vínculos entre o paciente e a família, tendo como norte refazer os laços entre o homem e seu ambiente/universo/ seu grupo social.

\section{A FAMÍLIA E A SAÚDE DA FAMÍLIA}

Quando passamos a considerar o usuário inserido em contexto familiar, passamos também a 
ver a família como usuária e como tal merecendo todo o esforço para entendê-la e prestar um cuidado eficiente. Assim, a enfermagem descobre que a família não é recipiente passivo do cuidado profissional, mas agente e sujeito do seu próprio processo de viver. Portanto o cuidar da família é processo complexo, pois envolve compreendê-la em sua totalidade ${ }^{4}$.

Embora o conceito de família seja muito subjetivo, cabe aqui colocar o conceito com o que mais nos identificamos: "É uma unidade dinâmica, constituída por pessoas que se percebem como família, com certo tempo de convívio, constituindo uma história de vida. Os membros estão unidos por laços de adoção, afetividade ou consangüinidade" 5:92 .

Acreditamos que o equilíbrio familiar pode ser abalado em situações de doença de algum de seus componentes, principalmente quando se refere a uma doença grave ou de doença crônica. Novos papéis precisam ser definidos, o estresse ronda a família. Cabe a equipe interdisciplinar trabalhar de forma que este equilíbrio não seja desfeito mas, eventualmente, que seja recuperado.

Acrescentamos a esta compreensão do que se entende por família saudável:

É uma unidade de auto-estima positiva, onde os membros convivem e se percebem mutuamente como família. Tem estrutura e organização para definir objetivos e promover os meios para o crescimento, desenvolvimento, saúde e bem-estar de seus membros. A família saudável se une por laços de afetividade exteriorizados por amor e carinho, tem liberdade de expor sentimentos e dúvidas, compartilha crenças, valores e conhecimentos. Aceita a individualidade de seus membros, possui capacidade de conhecer e usufruir seus direitos, enfrenta crises, conflitos e contradições, pedindo e dando apoio a seus membros e às pessoas significativas. A família saudável atua conscientemente no ambiente em que vive, interagindo dinamicamente com outras pessoas e famílias em diversos níveis de aproximação, transformando e sendo transformada. Desenvolve-se com experiência construindo sua história de vida 6:67.

O que muitas vezes encontramos na realidade são famílias doentes que já não conseguem atuar como unidades de saúde para seus membros e é neste momento que percebemos a importância do profissional que consegue perceber e trabalhar com este fato ou que consegue também trazer informações no sentido de prevenir agravos quando se trata de família saudável.

Nesses contatos as relações interpessoais dos profissionais, cliente e família se fortalecem cada vez mais; e nisso criam-se vínculos que fazem com que os familiares adquiram maior segurança $e$ confiança, respeitando a equipe. Permitem ainda maior aceitação da família em relação às suas responsabilidades de tratamento e de cuidado o que é, sem dúvida, fator positivo na recuperação da saúde do cliente ${ }^{6}$.

Para se aproximar das famílias dentro de uma abordagem sistêmica ${ }^{7}$, pactuamos que se aplicaria o genograma de três gerações e o ecomapa, somente em situações em que houvesse concordância e nas indicações de famílias em risco ou que houvesse dificuldade de ação da equipe de saúde depois de melhora do quadro crítico que gerou a atenção e intervenção da equipe de saúde.

\section{OBJETIVOS DO PROJETO DE ATUAÇÃO EM CUIDADO DOMICILIAR}

Promover cuidados de saúde por meio da assistência domiciliar nas áreas de atuação das unidades básicas de saúde da UFPEL; implantar o projeto de cuidados domiciliares nas UBS da UFPEL; implantar o projeto de cuidados domiciliares nas UBS da UFPEL; identificar e acompanhar sistematicamente a população das áreas delimitadas das UBS da UFPEL que não comparecem ao serviço por limitações físicas, psíquicas ou sociais; estabelecer estratégias para a periodicidade na marcação das visitas domiciliares conforme a estratificação de risco e necessidades de cuidados domiciliares; propor ações conjuntas com as demais ações programáticas da UBS; instrumentalizar os pacientes e familiares quanto a ações de auto-cuidado conforme a viabilidade e as necessidades; introduzir e estabelecer rotinas que facilitem e otimizem a realização dos cuidados 
quando é solicitada a visita domiciliar: eis a lista dos principais objetivos de atuação no concernente ao cuidado domiciliar.

\section{METODOLOGIA DE TRABALHO}

\section{INGRESSO NO PROGRAMA}

Serão inscritos, inicialmente, os usuários das áreas de abrangência das unidades que já recebem algum tipo de assistência, fora do ambiente físico dessas unidades e, posteriormente, serão inscritos aqueles usuários que contemplam os critérios de inclusão do programa. Poderão ser inscritos também os usuários que solicitam visita domiciliar e que estejam necessitando de cuidados domiciliares.

O ingresso no Programa será estabelecido pela equipe multidisciplinar. A freqüência das visitas, que serão individualizadas e padronizadas em cuidados diários, semanais, quinzenais e mensais, poderá ser alterada conforme a necessidade do usuário.

\section{MANUTENÇÃO DO PROGRAMA}

A partir da solicitação da primeira visita (Anexo 1) ou de algum cuidado fora do calendário estabelecido, a equipe de saúde definira quando este cuidado ocorrerá, (no formulário de solicitação) e encaminhará ao responsável pelo cuidado: médico, enfermeiro, residente, acadêmicos de enfermagem e de medicina.

Cada paciente em sua primeira visita receberá explicações objetivas sobre o programa e o nome do profissional responsável pelo seu atendimento, assim como o telefone da UBS, além da freqüência das visitas com data e hora marcadas.

Na UBS ficará o registro (Ficha de Cuidado Domiciliar- Anexo 2) do paciente, em lugar específico (arquivo), onde haverá, ainda, um calendário de todas as visitas, a ser elaborado pelo responsável do programa.

Há necessidade da elaboração de rotinas para a solicitação de visitas domiciliares para agilizar atendimento àqueles pacientes que não estão incluídos no programa.
Estão previstas reuniões trimestrais com as famílias, com estes objetivos: 1) Ajudar o cuidador e outros familiares para que estes longe de seu parente doente exponham suas dificuldades, ansiedades, dúvidas e o que mais desejassem. 2) Compartilhar os problemas entre os familiares para visualizar seu caráter comum. A partir das necessidades, podem-se realizar propostas de trabalho, como capacitações para cuidadores, etc.

O responsável pelo programa divulgará um calendário para as visitas do mês previstas na Ficha de Cuidado Domiciliar.

Reuniões quinzenais previamente determinadas deverão ser realizadas com equipe multidisciplinar da UBS, para a discussão do programa e avaliação.

Todos os profissionais da equipe poderão realizar atendimento domiciliar, respeitando-se as necessidades do paciente e o membro da equipe responsável pelo paciente.

\section{POPULAÇÃO ALVO}

A população alvo deste projeto são as pessoas residentes nas áreas de abrangência das unidades básicas de saúde da UFPE e que estejam de acordo com o perfil dos critérios de inclusão no programa.

Critérios de inclusão no programa

- População idosa com limitações físicas que impossibilitem seu deslocamento à unidade de saúde.

- Indivíduos que apresentem seqüelas, devido a acidente vascular cerebral; portadores de limitações físicas, visuais e/ou psíquicas; indivíduos com patologias agudas que, após avaliação da equipe interdisciplinar, possam ser beneficiados com os cuidados domiciliares; pacientes em fase terminal, portadores de neoplasia maligna; com presença de ferida cirúrgica ou úlceras de pressão; debilitados que necessitem de medicação (I.V., I.M., OU V.O.), ou treinamento para a sua administração (insulinoterapia, antibioticoterapia); indivíduos que apresentem dificuldades para comparecer à UBS para consultas periódicas, dificuldade de locomoção, amputações, fraturas. 
- Possuir familiares ou responsáveis (cuidador), com disponibilidade, física e psicológica, para participação no programa de assistência domiciliar em caráter transitório.

- Indivíduo que necessita de vigilância de educação em saúde, por infestação de pediculose, doença infecto-contagiosa.

Critérios de desligamento do programa

- Melhora no quadro, ou reversão da condição incapacitante.

- Mudança da área de responsabilidade da UBS.

- Solicitação do paciente e/ou familiar, para o não recebimento dos cuidados oferecidos, por meio de manifestação oral e registro no prontuário.

- Necessidade de institucionalização.

- Óbito.

\section{AVALIAÇÃO DO PROJETO}

A avaliação dos serviços de saúde, adaptada às necessidades do cuidado domiciliar seguirá a metodologia adotada em outros programas ${ }^{8}$, especificando-se três categorias: indicadores de estrutura, processo e resultado.

\section{$\sqrt{ }$ Indicadores Estrutura}

$\sqrt{ }$ Número total de inscritos

$\sqrt{ }$ Número de visitas previstas/mês

$\sqrt{ }$ Número de visitas realizadas/mês

$\sqrt{ }$ Profissionais disponíveis para o desenvolvimento do projeto

\section{$\sqrt{ }$ Indicadores Processo}

$\sqrt{ }$ Intercorrências/Mês (Visitas fora do calendário previsto).

$\sqrt{ }$ Internações/Mês

$\sqrt{ }$ Encaminhamentos/Mês

$\sqrt{ }$ Indicadores Resultados

Instrumento de avaliação da qualidade de vida reduzido - WHOQOL ${ }^{9}$, indicado pela Organização Mundial de Saúde.

\section{CONSIDERAÇÕES FINAIS}

A elaboração desta proposta foi uma experiência ímpar para o grupo que organizou as discussões e o roteiro; durante a sua estruturação, houve participação de diferentes profissionais que atuavam nas equipes, como assistente social, estudantes de graduação em enfermagem e medicina. Além disso, o grupo necessitou fazer algumas opções em relação às bibliografias, o que gerou discussões importantes dentro de um prazo temporal, pois se trabalhou com um limite determinado para iniciar a implantação do projeto.

A avaliação da equipe responsável pelo programa foi muito boa e produtiva, recomenda-se que seja implementada e aperfeiçoada no decorrer do cuidado domiciliar. As sugestões foram de socializar a proposta a fim de que se formule uma política em torno do tema e que se desenvolvam pesquisas para complementar metodologias de cuidado domiciliar.

\section{REFERÊNCIAS}

1 Luz MT. Políticas de descentralização e cidadania: novas práticas em saúde no Brasil atual. In: Pinheiro R, Mattos RA. Os sentidos da integralidade na atenção e no cuidado à saúde. Rio de Janeiro: ABRASCO; 2001.

2 Kleinman A. Conceps and model for the comparison of medical systems as cultural Systems. Soc Sc Med 1980; 12:83-93.

3 Colleère MF. Promover a vida. Lisboa: Lidel; 1999. p.32.

4 Elsen I et al., organizadores. Marcos para a prática de Enfermagem com famílias. Florianópolis: Ed. da UFSC; 1994.

5 Beck CLC. Sofrimento e esperança - Vivências com familiares de pacientes internados em UTI. In: Gonzales RMG, organizador. Cenários de cuidado. Santa Maria: Pallotti;1999.p.61-155.

6 Cargin MT, Costenaro RTS, Lacerda MR. Famíliaenfermeira: Uma relação necessária na UTI In:Costenaro RS, et al. Cuidando em enfermagem: pesquisas e reflexões. Santa Maria: Centro Universitário Franciscano; 2001.p.15-34; 67.

7 Wright LM, Leahey M. Enfermeiras e famílias: um guia para avaliação e intervenção na família. São Paulo: Roca; 2002.

8 Donabedian A. The quality of car: how can it be assessed? JAMA, 1984; 260(12): 1734-48.

9 The WHOQOL Group. The World Health Organization quality of life assessment (WHOQOL): position paper from the World Health Orgnization. Soc Sc Méd 1995; 10: p. 1403-9. 
ANEXO 1

SOLICITAÇÃO DE VISITA DOMICILIAR

Nome:

Idade:

Data:

No Prontuário:

Queixa principal:

Sinais e sintomas:

$\begin{array}{ll}\text { ( ) Falta de ar } & (\quad) \text { Tosse com escarro } \\ (\quad) \text { Febre } & (\quad) \text { Dor } \\ (\quad) \text { Diarréia } & (\quad) \text { Vômitos/Náuseas } \\ & \end{array}$




\section{ANEXO 2}

FICHA DE CUIDADOS DOMICILIARES

\begin{tabular}{l|l|l|}
\hline NOME: & DATA NASC: & NR PRONTUÁRIO: \\
\hline ENDEREÇO: & TELEFONE: \\
\hline
\end{tabular}

PROBLEMAS/ENFERMIDADES:

CRITÉRIO(S) DE INCLUSÃO:

CONDIÇÃO INCAPACITANTE PRINCIPAL:

MEDICAÇÃO UTILIZADA:

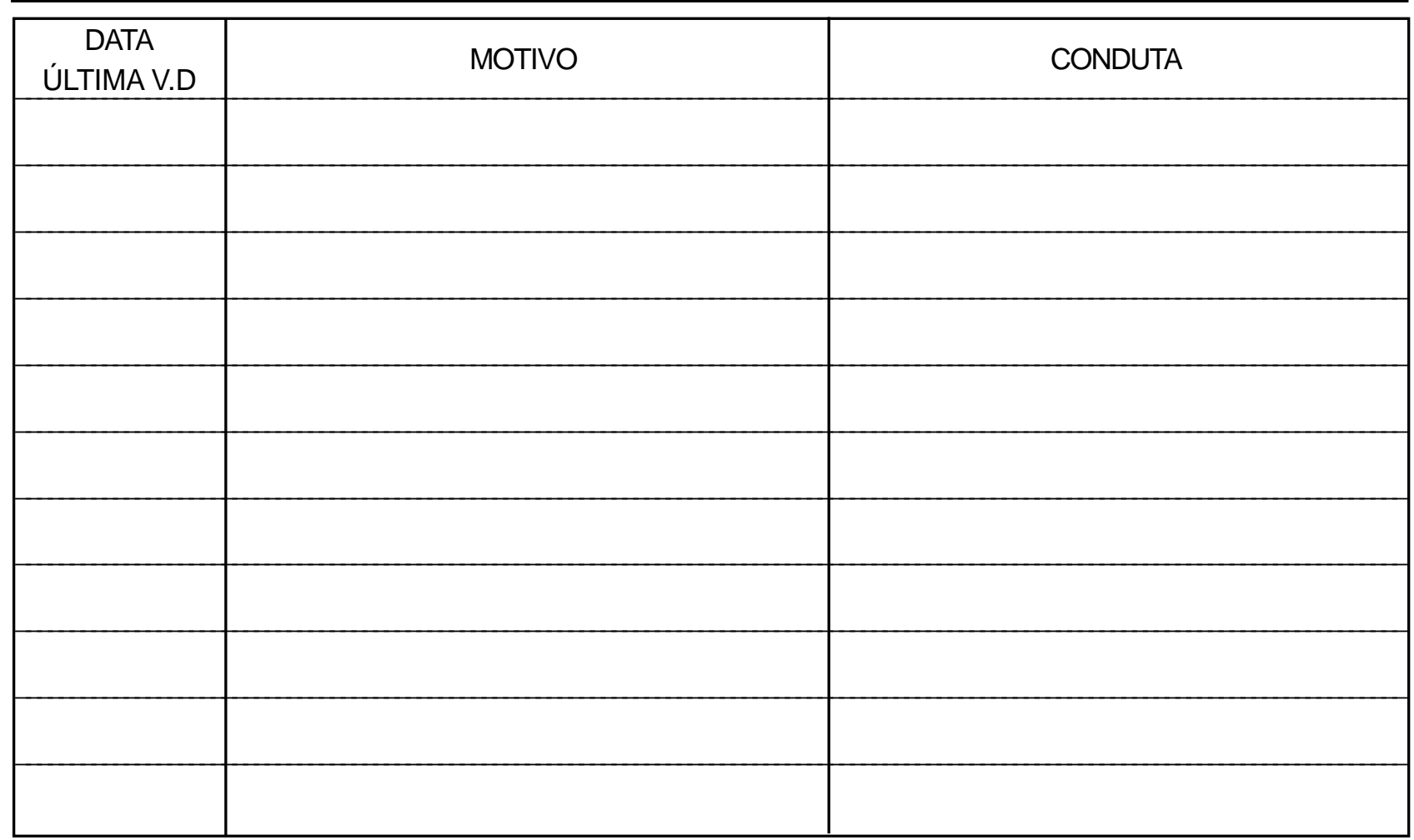


INTERNAÇÕES HOSPITALARES

\begin{tabular}{|l|l|}
\hline DATA & \\
\hline & \\
\hline & \\
\hline & \\
\hline & \\
\hline & \\
\hline & \\
\hline & \\
\hline & \\
\hline
\end{tabular}

ENCAMINHAMENTOS

\begin{tabular}{|l|l|l|}
\hline DATA & LOCAL & \\
\hline & & \\
\hline & & \\
\hline & & \\
\hline & & \\
\hline & & \\
\hline & & \\
\hline & & \\
\hline & & \\
\hline & & \\
\hline
\end{tabular}

EXCLUSÃO DO PROGRAMA

\begin{tabular}{|c|c|}
\hline DIA & CRITÉRIO DE EXCLUSÃO \\
\hline & \\
\hline
\end{tabular}

OBSERVAÇÕES: 